\title{
Erratum: \\ Codes of Steiner Triple and Quadruple Systems Designs, Codes and Cryptography, 3, 117-125 \\ (1993)
}

On page 122, the end of the second line of Proposition 2 should be altered to read

"Then $C^{\perp} \subseteq C$ unless $p=3,9$ does not divide $v$, and $\mathcal{D}$ has precisely three (disjoint) affine hyperplanes."

On page 123, in the proof, the second line should be altered to read

" $w=v^{S_{1}}-v^{S_{-1}} \in C$ (unless 3 does not divide $v / 3$ ), and $C^{\perp} \subseteq C$." 